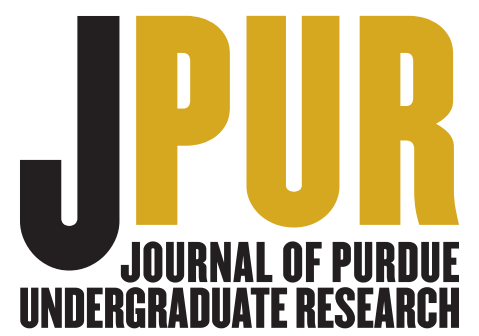

\title{
STEM
}

\section{Influence of 3D Printing Infill Patterns on the Effective Thermal Properties}

\section{Student researcher: Yu Han, Senior}

The interior of most 3D printed objects is not completely solid, but rather a partially hollow infill pattern is used to save material and manufacturing time. Most commonly used infill designs were optimized for the mechanical strengthto-weight ratio, but little research exists on the effect of the 3D printed infill pattern on the thermal conductivity. As 3D printing penetrates into all types of industries and research areas, thermal properties become increasingly important.

This project focuses on characterizing the effective thermal conductivity of 3D printed parts with different infill patterns. Specifically, three major commercially used infilled patterns (zigzag, linear crossing, and hexagon) are investigated experimentally and through numerical simulations. The thermal conductivity of $3 \mathrm{D}$ printed parts is not expected to be isotropic, and the orientation of the 3D printing filaments impacts the heat conduction anisotropy.

Thermal models are first used to study the impact of infill pattern parameters on thermal conductivity, $k$. Neglecting natural convection, the models predict that thermal conductivity depends nearly linearly on the volume infill percentage, but the pattern orientation also impacts heat transfer. For the zig-zag infill patterns, as the infill supports are rotated, the highest $k$ occurs when the supports are aligned with the direction of heat flow, and the lowest $k$ occurs when the supports are perpendicular to the heat flow direction.

Experiments on 3D printed samples using a comparative IR microscopy technique indicate good agreement with the trends predicted by the simulations. The major discrepancy between simulated and experimental results is due to natural convection.
In future research, the experiment will be improved to probe the effect of convection and the filament diameter on the thermal conductivity.

Research advisor Amy Marconnet writes: "As we engineer new devices taking advantage of the unique capabilities of $3 D$ printing, understanding the multifunctional performance of these materials is crucial. Yu's research is the first step toward optimizing the thermal and mechanical properties of $3 D$ printed structures." (a)

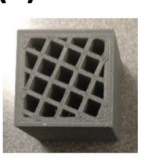

Linear Crossing

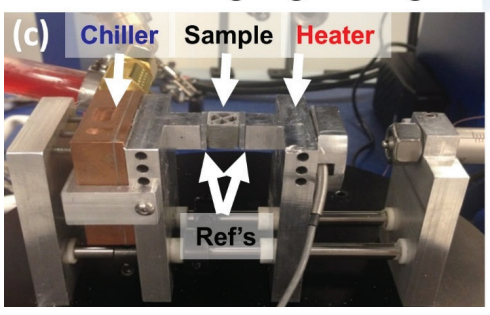

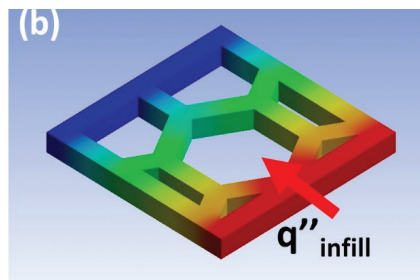

(d)

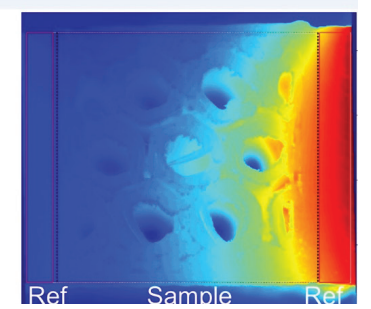

(a) 3D printed samples with linear crossing, zig-zag, and hexagonal infill patterns. (b) Simulated temperature map through the hexagonal infill pattern. (c) Experimental setup: A heater generates heat that conducts through the sample to the chiller/cold plate. The reference layers on either side of the sample quantification of heat flow through the sample. (d) Experimentally measured temperature map for the referencesample-reference stack. 